\title{
On the Heyde theorem for discrete Abelian groups
}

\author{
by
}

\author{
G. M. Feldman (Kharkov)
}

\begin{abstract}
Let $X$ be a countable discrete Abelian group, $\operatorname{Aut}(X)$ the set of automorphisms of $X$, and $I(X)$ the set of idempotent distributions on $X$. Assume that $\alpha_{1}, \alpha_{2}, \beta_{1}, \beta_{2} \in \operatorname{Aut}(X)$ satisfy $\beta_{1} \alpha_{1}^{-1} \pm \beta_{2} \alpha_{2}^{-1} \in \operatorname{Aut}(X)$. Let $\xi_{1}, \xi_{2}$ be independent random variables with values in $X$ and distributions $\mu_{1}, \mu_{2}$. We prove that the symmetry of the conditional distribution of $L_{2}=\beta_{1} \xi_{1}+\beta_{2} \xi_{2}$ given $L_{1}=\alpha_{1} \xi_{1}+\alpha_{2} \xi_{2}$ implies that $\mu_{1}, \mu_{2} \in I(X)$ if and only if the group $X$ contains no elements of order two. This theorem can be considered as an analogue for discrete Abelian groups of the well-known Heyde theorem where the Gaussian distribution on the real line is characterized by the symmetry of the conditional distribution of one linear form given another.
\end{abstract}

1. Introduction. The well-known Skitovich-Darmois theorem asserts that a Gaussian distribution on the real line is characterized by the independence of two linear forms of independent random variables. A similar result of Heyde characterizes a Gaussian distribution by the symmetry of the conditional distribution of one linear form given another.

Theorem A (C. C. Heyde [6], see also [7, §13.4]). Let $\xi_{1}, \ldots, \xi_{n}, n \geq 2$, be independent random variables, and let $\alpha_{j}, \beta_{j}$ be nonzero constants such that $\beta_{i} \alpha_{i}^{-1} \pm \beta_{j} \alpha_{j}^{-1} \neq 0$ for all $i \neq j$. If the conditional distribution of $L_{2}=\beta_{1} \xi_{1}+\cdots+\beta_{n} \xi_{n}$ given $L_{1}=\alpha_{1} \xi_{1}+\cdots+\alpha_{n} \xi_{n}$ is symmetric, then all random variables $\xi_{j}$ are Gaussian.

Let $X$ be a locally compact Abelian separable metric group. Denote by $Y=X^{*}$ the character group of $X$. Let $(x, y)$ be the value of a character $y \in Y$ on an element $x \in X$. Denote by $M^{1}(X)$ the convolution semigroup of probability distributions on $X$. For $\mu \in M^{1}(X)$ denote by $\widehat{\mu}$ its characteristic function,

$$
\widehat{\mu}(y)=\int_{X}(x, y) d \mu(x) .
$$

2000 Mathematics Subject Classification: Primary 62E10; Secondary 60B15, 39B52.

Key words and phrases: discrete Abelian group, characterization of probability distributions, idempotent distribution. 
A distribution $\mu \in M^{1}(X)$ is called Gaussian ([9, Ch. IV]) if its characteristic function can be represented in the form

$$
\widehat{\mu}(y)=(x, y) \exp \{-\varphi(y)\}, \quad y \in Y,
$$

where $x \in X$ and $\varphi$ is a continuous nonnegative function on $Y$ satisfying the equation

$$
\varphi(u+v)+\varphi(u-v)=2[\varphi(u)+\varphi(v)], \quad u, v \in Y .
$$

Let $\operatorname{Aut}(X)$ be the set of topological automorphisms of $X$, and let $\xi_{1}, \ldots, \xi_{n}$, $n \geq 2$, be independent random variables with values in $X$ and distributions $\mu_{j}$. Consider the linear forms $L_{1}=\alpha_{1} \xi_{1}+\cdots+\alpha_{n} \xi_{n}$ and $L_{2}=\beta_{1} \xi_{1}+$ $\cdots+\beta_{n} \xi_{n}$, where $\alpha_{j}, \beta_{j} \in \operatorname{Aut}(X)$ satisfy the condition $\beta_{i} \alpha_{i}^{-1} \pm \beta_{j} \alpha_{j}^{-1} \in$ $\operatorname{Aut}(X)$ for all $i \neq j$. Let us formulate the following general problem.

Problem 1. Describe locally compact Abelian separable metric groups $X$ for which the symmetry of the conditional distribution of $L_{2}=\beta_{1} \xi_{1}+$ $\cdots+\beta_{n} \xi_{n}$ given $L_{1}=\alpha_{1} \xi_{1}+\cdots+\alpha_{n} \xi_{n}$ implies that all distributions $\mu_{j}$ are either Gaussian or belong to a class of distributions that can be considered as a natural analogue of the class of Gaussian distributions.

Problem 1 was solved in the class of finite Abelian groups ([3]) and in the class of all locally compact Abelian separable metric groups under the additional assumption that the characteristic functions of the distributions $\mu_{j}$ do not vanish ([4]). The aim of the article is to give the solution of Problem 1 in the class of countable discrete Abelian groups. We will also study some similar problems.

We shall first fix some notation. If $G$ is a subgroup of $X$, then denote by $A(Y, G)=\{y \in Y:(x, y)=1$ for all $x \in G\}$ its annihilator. For $\alpha \in \operatorname{Aut}(X)$ we define the conjugate automorphism $\widetilde{\alpha} \in \operatorname{Aut}(Y)$ by the formula $(x, \widetilde{\alpha} y)=(\alpha x, y)$ for all $x \in X, y \in Y$. Denote by $I$ the identity automorphism of a group. Let $f_{2}: X \rightarrow X$ be the homomorphism $f_{2} x=2 x$ and put $X_{(2)}=\operatorname{Ker} f_{2}, X^{(2)}=\operatorname{Im} f_{2}$. Denote by $\mathbb{T}$ the circle group (the one-dimensional torus) and by $\mathbb{Z}$ the group of integers. If $A$ and $B$ are subsets of $Y$, denote by $A+B=\{y \in Y: y=u+v, u \in A, v \in B\}$ their arithmetic sum. Let $\psi$ be an arbitrary function on $Y$ and let $h \in Y$. Denote by $\Delta_{h}$ the finite difference operator

$$
\Delta_{h} \psi(y)=\psi(y+h)-\psi(y), \quad y \in Y .
$$

A continuous function $\psi$ on $Y$ is called a polynomial if for some nonnegative integer $m$,

$$
\Delta_{h}^{m+1} \psi(y)=0 \quad \text { for all } y, h \in Y .
$$

If $\xi$ is a random variable with values in $X$ and with distribution $\mu$, then $\widehat{\mu}(y)=\mathbf{E}[(\xi, y)]$. For $\mu \in M^{1}(X)$ we define $\bar{\mu} \in M^{1}(X)$ by $\bar{\mu}(E)=\mu(-E)$ for all Borel sets $E \subset X$. Observe that $\widehat{\mu}(y)=\overline{\widehat{\mu}}(y)$. Denote by $E_{x}$ the 
degenerate distribution concentrated at a point $x \in X$, and by $\sigma(\mu)$ the support of $\mu \in M^{1}(X)$. Let $I(X)$ be the set of idempotent distributions on $X$, i.e. the set of shifts of the Haar distributions $m_{K}$ of compact subgroups $K$ of $X$. Note that

$$
\widehat{m}_{K}(y)= \begin{cases}1, & y \in A(Y, K) \\ 0, & y \notin A(Y, K)\end{cases}
$$

Observe that the Gaussian distributions on a discrete Abelian group $X$ are degenerate, and the class $I(X)$ can be regarded as a natural analogue of the class of Gaussian distributions for discrete Abelian groups. We remark that if $H$ is a closed subgroup of $Y$ and $\widehat{\mu}(y)=1$ for $y \in H$, then $\widehat{\mu}$ is $H$-invariant, i.e. $\widehat{\mu}(y+h)=\widehat{\mu}(y)$ for all $y \in Y, h \in H$, and $\sigma(\mu) \subset A(X, H)$. We will use the well-known facts concerning the structure of locally compact Abelian groups and the duality theory (see [5]). We now formulate the main result of the article.

Theorem 1. Let $X$ be a countable discrete Abelian group. Assume that $\alpha_{1}, \alpha_{2}, \beta_{1}, \beta_{2} \in \operatorname{Aut}(X)$ satisfy $\beta_{1} \alpha_{1}^{-1} \pm \beta_{2} \alpha_{2}^{-1} \in \operatorname{Aut}(X)$. Let $\xi_{1}, \xi_{2}$ be independent random variables with values in $X$ and distributions $\mu_{1}, \mu_{2}$. The symmetry of the conditional distribution of $L_{2}=\beta_{1} \xi_{1}+\beta_{2} \xi_{2}$ given $L_{1}=\alpha_{1} \xi_{1}+\alpha_{2} \xi_{2}$ implies that $\mu_{1}, \mu_{2} \in I(X)$ if and only if $X_{(2)}=\{0\}$, i.e. the group $X$ contains no elements of order two.

First we study the case when $X$ is a discrete torsion-free Abelian group.

\section{The Heyde theorem for discrete torsion-free Abelian groups.}

We will prove the group analogue of the Heyde theorem for discrete torsionfree Abelian groups and use this result to prove Theorem 1. We need some lemmas.

Lemma 1 ([4]). Let $X$ be a locally compact Abelian separable metric group. Let $\xi_{1}, \ldots, \xi_{n}, n \geq 2$, be independent random variables with values in $X$ and distributions $\mu_{j}$. Assume that $\alpha_{j}, \beta_{j} \in \operatorname{Aut}(X)$. The conditional distribution of $L_{2}=\beta_{1} \xi_{1}+\cdots+\beta_{n} \xi_{n}$ given $L_{1}=\alpha_{1} \xi_{1}+\cdots+\alpha_{n} \xi_{n}$ is symmetric if and only if

$$
\prod_{j=1}^{n} \widehat{\mu}_{j}\left(\widetilde{\alpha}_{j} u+\widetilde{\beta}_{j} v\right)=\prod_{j=1}^{n} \widehat{\mu}_{j}\left(\widetilde{\alpha}_{j} u-\widetilde{\beta}_{j} v\right), \quad u, v \in Y .
$$

Lemma 2 ([1], see also [2, Appendix 1]). Let $Y$ be a compact Abelian group and $\psi(y)$ be a polynomial on $Y$. Then $\psi(y)=$ const.

Lemma $3([8$, Ch. $6, \S 1])$. Let $F(t), t \in \mathbb{R}^{k}$, be a characteristic function, and let $\Phi(t), t \in \mathbb{R}^{k}$, be the restriction to $\mathbb{R}^{k}$ of an entire function $\Phi(z)$, $z \in \mathbb{C}^{k}$. Assume that

$$
F(t)=\Phi(t), \quad t \in U,
$$


where $U$ is a neighbourhood of zero in $\mathbb{R}^{k}$. Then $F(t)$ can be extended onto $\mathbb{C}^{k}$ as an entire function and (2) holds for all $\mathbb{R}^{k}$.

We can now prove the main result of this section.

Proposition 1. Let $X$ be a countable discrete torsion-free Abelian group. Assume that $\alpha_{j}, \beta_{j} \in \operatorname{Aut}(X)$ satisfy $\beta_{i} \alpha_{i}^{-1} \pm \beta_{j} \alpha_{j}^{-1} \in \operatorname{Aut}(X)$ for all $i \neq j$. Let $\xi_{1}, \ldots, \xi_{n}, n \geq 2$, be independent random variables with values in $X$ and distributions $\mu_{j}$. If the conditional distribution of $L_{2}=$ $\beta_{1} \xi_{1}+\cdots+\beta_{n} \xi_{n}$ given $L_{1}=\alpha_{1} \xi_{1}+\cdots+\alpha_{n} \xi_{n}$ is symmetric, then all $\mu_{j}$ are degenerate distributions.

Proof. By Lemma 1, the symmetry of the conditional distribution of $L_{2}$ given $L_{1}$ implies that the characteristic functions $\widehat{\mu}_{j}$ satisfy (1). We note that $Y=X^{*}$ is a connected compact Abelian group. Passing to the random variables $\xi_{j}^{\prime}=\alpha_{j} \xi_{j}$ we can assume without loss of generality that $L_{1}=$ $\xi_{1}+\cdots+\xi_{n}$ and $L_{2}=\delta_{1} \xi_{1}+\cdots+\delta_{n} \xi_{n}$, where the automorphisms $\delta_{j} \in \operatorname{Aut}(X)$ satisfy $\delta_{i} \pm \delta_{j} \in \operatorname{Aut}(X)$ for all $i \neq j$. Then equation (1) is transformed into

$$
\prod_{j=1}^{n} \widehat{\mu}_{j}\left(u+\widetilde{\delta}_{j} v\right)=\prod_{j=1}^{n} \widehat{\mu}_{j}\left(u-\widetilde{\delta}_{j} v\right), \quad u, v \in Y,
$$

where $\widetilde{\delta}_{i} \pm \widetilde{\delta}_{j} \in \operatorname{Aut}(Y)$ for all $i \neq j$. It is clear that the characteristic functions of the distributions $\nu_{j}=\mu_{j} * \bar{\mu}_{j}$ also satisfy (3). Set $f_{j}(y)=\widehat{\nu}_{j}(y)$, $\varepsilon_{j}=\widetilde{\delta}_{j}$ and rewrite equation (3) using the new notation:

$$
\prod_{j=1}^{n} f_{j}\left(u+\varepsilon_{j} v\right)=\prod_{j=1}^{n} f_{j}\left(u-\varepsilon_{j} v\right), \quad u, v \in Y .
$$

We will prove that $f_{j}(y)=1$ for all $y \in Y$ and $j$. It is obvious that $f_{j}(y)=$ $\left|\widehat{\mu}_{j}(y)\right|^{2} \geq 0$. Choose a neighbourhood $U$ of zero in $Y$ such that $f_{j}(y)>0$ for all $y \in U$ and $j$. Set $\psi_{j}(y)=-\ln f_{j}(y), y \in U$. Take a symmetric neighbourhood $U_{1}$ of zero in $Y$ such that $U_{1}+\varepsilon_{j}\left(U_{1}\right) \subset U, j=1, \ldots, n$. The functions $\psi_{j}$ satisfy

$$
\sum_{j=1}^{n} \psi_{j}\left(u+\varepsilon_{j} v\right)=\sum_{j=1}^{n} \psi_{j}\left(u-\varepsilon_{j} v\right), \quad u, v \in U_{1} .
$$

In order to solve this equation we apply the finite difference method. We restrict ourselves to the case $n=2$. Let $V$ be a symmetric neighbourhood of zero in $Y$ such that

$$
\sum_{j=1}^{8} \lambda_{j}(V) \subset U
$$

for any $\lambda_{j} \in\left\{I, \varepsilon_{1}, \varepsilon_{2}\right\}$. Then

(4) $\psi_{1}\left(u+\varepsilon_{1} v\right)+\psi_{2}\left(u+\varepsilon_{2} v\right)-\psi_{1}\left(u-\varepsilon_{1} v\right)-\psi_{2}\left(u-\varepsilon_{2} v\right)=0, \quad u, v \in V$. 
Let $k_{1} \in V$. Put $h_{1}=\varepsilon_{2} k_{1}$ and hence $h_{1}-\varepsilon_{2} k_{1}=0$. Give $u$ and $v$ in (4) the increments $h_{1}$ and $k_{1}$ respectively. Subtracting (4) from the resulting equation we find

$$
\Delta_{l_{11}} \psi_{1}\left(u+\varepsilon_{1} v\right)+\Delta_{l_{12}} \psi_{2}\left(u+\varepsilon_{2} v\right)-\Delta_{l_{13}} \psi_{1}\left(u-\varepsilon_{1} v\right)=0, \quad u, v \in V,
$$

where $l_{11}=\left(\varepsilon_{2}+\varepsilon_{1}\right) k_{1}, l_{12}=2 \varepsilon_{2} k_{1}, l_{13}=\left(\varepsilon_{2}-\varepsilon_{1}\right) k_{1}$. Let $k_{2} \in V$. Put $h_{2}=\varepsilon_{1} k_{2}$ and hence $h_{2}-\varepsilon_{1} k_{2}=0$. Give $u$ and $v$ in (5) the increments $h_{2}$ and $k_{2}$ respectively. Subtracting (5) from the resulting equation we arrive at

$$
\Delta_{l_{21}} \Delta_{l_{11}} \psi_{1}\left(u+\varepsilon_{1} v\right)+\Delta_{l_{22}} \Delta_{l_{12}} \psi_{2}\left(u+\varepsilon_{2} v\right)=0, \quad u, v \in V,
$$

where $l_{21}=2 \varepsilon_{1} k_{2}, l_{22}=\left(\varepsilon_{1}+\varepsilon_{2}\right) k_{2}$. Let $k_{3} \in V$. Put $h_{3}=-\varepsilon_{2} k_{3}$ and hence $h_{3}+\varepsilon_{2} k_{3}=0$. Give $u$ and $v$ in (6) the increments $h_{3}$ and $k_{3}$ respectively. Subtracting (6) from the resulting equation we find

$$
\Delta_{l_{31}} \Delta_{l_{21}} \Delta_{l_{11}} \psi_{1}\left(u+\varepsilon_{1} v\right)=0, \quad u, v \in V,
$$

where $l_{31}=\left(\varepsilon_{1}-\varepsilon_{2}\right) k_{3}$. Substituting $v=0$ in (7) we infer that

$$
\Delta_{l_{31}} \Delta_{l_{21}} \Delta_{l_{11}} \psi_{1}(u)=0, \quad u \in V .
$$

Since $Y$ is a connected Abelian group, we have $Y^{(2)}=Y$. Hence, $f_{2}: Y \rightarrow Y$ is an open homomorphism. The condition $\varepsilon_{1} \pm \varepsilon_{2} \in \operatorname{Aut}(Y)$, the expressions for $l_{11}, l_{21}, l_{31}$ and equation (8) imply that there is a neighbourhood $W$ of zero in $Y$ such that

$$
\Delta_{h}^{3} \psi_{1}(y)=0, \quad h, y \in W .
$$

Since $Y$ is a connected compact Abelian group, this implies that there exists a compact subgroup $H \subset W$ such that $Y / H \approx \mathbb{T}^{k}([5, \S 24.7])$. Consider the restriction of equation (9) to $H$. As by Lemma 2 , all polynomials on a compact Abelian group are constants and $\psi_{1}(0)=0$, we have $\psi_{1}(y)=0$, $y \in H$. Hence, $f_{1}(y)=1, y \in H$. It follows that $f_{1}(y+h)=f_{1}(y), y \in Y$, $h \in H$. Let $p_{1}: Y \rightarrow Y / H$ be the natural homomorphism, and $p_{2}: Y / H \rightarrow$ $\mathbb{T}^{k}$ be the above mentioned isomorphism. Consider the composition $p=$ $p_{2} p_{1}: Y \rightarrow \mathbb{T}^{k}$. Since $p$ is an open homomorphism, $p(W)$ is a neighbourhood of zero in $\mathbb{T}^{k}$. Denote elements of $\mathbb{T}^{k}$ by $t=\left(t_{1}, \ldots, t_{k}\right)$, where $-\pi \leq t_{j}$ $<\pi$. The group operation in $\mathbb{T}^{k}$ is coordinatewise addition modulo $2 \pi$. The function $f_{1}$ induces a positive definite function $\widetilde{f}_{1}$ on $\mathbb{T}^{k}$ by the formula $\widetilde{f}_{1}(t)=f_{1}(y), t=p y$. By the Bochner theorem, there is a distribution $\lambda_{1} \in M^{1}\left(\mathbb{Z}^{k}\right)$ such that $\widehat{\lambda}_{1}(t)=\widetilde{f}_{1}(t), t \in \mathbb{T}^{k}$. Moreover it follows from (9) that in the neighbourhood $p(W)$ of zero in $\mathbb{T}^{k}$ we have the representation

$$
\widetilde{f}_{1}(t)=e^{-\widetilde{\psi}_{1}(t)}, \quad t \in p(W),
$$

where $\widetilde{\psi}_{1}(t)=\psi_{1}(y), t=p y$. It is clear that $\widetilde{\psi}_{1}(t)$ is an ordinary polynomial of $k$ variables. Since $\mathbb{Z}^{k} \subset \mathbb{R}^{k}$, we can consider $\lambda_{1}$ as a distribution on $\mathbb{R}^{k}$ 
with support in $\mathbb{Z}^{k}$, i.e. we can assume that the function $\widetilde{f}_{1}(t)$ is defined on $\mathbb{R}^{k}$ and is $2 \pi$-periodic in each variable. Note that the right hand side of (10) can be extended to $\mathbb{C}^{k}$ as an entire function. By Lemma 3, the same holds for the left-hand side of (10), and (10) holds for any $t \in \mathbb{R}^{k}$. Since the polynomial $\widetilde{\psi}_{1}(t)$ is $2 \pi$-periodic in each variable, we infer that $\widetilde{\psi}_{1}(t)=0$, $t \in \mathbb{R}^{k}$. This implies that $\widetilde{f}_{1}(t)=1, t \in \mathbb{R}^{k}$, and hence $f_{1}(y)=1, y \in Y$. We proved that $\nu_{1}$ is a degenerate distribution, so that the same is true for $\mu_{1}$. Reasoning similarly we prove that $\mu_{2}$ is also a degenerate distribution. The proof for arbitrary $n$ uses the same scheme. Proposition 1 is proved.

It is well known that any locally compact Abelian group is topologically isomorphic to a group of the form $\mathbb{R}^{m} \times G$, where $m \geq 0$ and $G$ contains a compact open subgroup. Proposition 1 implies the following statement.

Corollary 1. Assume that a locally compact Abelian separable metric group $X$ is of the form $X=\mathbb{R}^{m} \times G$, where $m \geq 0$ and the group $G$ contains a compact open subgroup. Assume that $\alpha_{j}, \beta_{j} \in \operatorname{Aut}(X)$. Let $\xi_{1}, \ldots, \xi_{n}, n \geq 2$, be independent random variables with values in $X$ and distributions $\mu_{j}$. If the conditional distribution of $L_{2}=\beta_{1} \xi_{1}+\cdots+\beta_{n} \xi_{n}$ given $L_{1}=\alpha_{1} \xi_{1}+\cdots+$ $\alpha_{n} \xi_{n}$ is symmetric, then for some shifts $\xi_{j}^{\prime}$ of the random variables $\xi_{j}$ the conditional distribution of $L_{2}^{\prime}=\beta_{1} \xi_{1}^{\prime}+\cdots+\beta_{n} \xi_{n}^{\prime}$ given $L_{1}^{\prime}=\alpha_{1} \xi_{1}^{\prime}+\cdots+\alpha_{n} \xi_{n}^{\prime}$ is symmetric and $\sigma\left(\mu_{j}^{\prime}\right) \subset \mathbb{R}^{m} \times G_{0}$ for all $j$, where $\mu_{j}^{\prime}$ is the distribution of $\xi_{j}^{\prime}$ and the subgroup $G_{0}$ consists of all compact elements of $G$.

Proof. Put $Y=X^{*}$ and denote by $C_{Y}$ the connected component of zero in $Y$. By the structure theorem for connected locally compact Abelian groups, $C_{Y}=M \times L$, where $M \approx \mathbb{R}^{m}$, and $L$ is a connected compact Abelian group. By Lemma 1, the symmetry of the conditional distribution of $L_{2}$ given $L_{1}$ is equivalent to equation (1). It is easily seen that $c(L)=L$ for any $c \in$ $\operatorname{Aut}(Y)$. Hence, we can restrict equation (1) to the subgroup $L$. Since $L$ is a connected compact Abelian separable metric group, it is the character group of a countable discrete torsion-free Abelian group. Proposition 1 implies that the restrictions of the characteristic functions $\widehat{\mu}_{j}$ to $L$ are characters of the subgroup $L$. Extending them to characters of $Y$ we find that there are $x_{j} \in X$ such that

$$
\widehat{\mu}_{j}(y)=\left(x_{j}, y\right), \quad y \in L, j=1, \ldots, n .
$$

Substitute (11) into (1) and consider the resulting equation on $L$. We infer that

$$
2 \sum_{j=1}^{n} \beta_{j} x_{j} \in A(X, L)=\mathbb{R}^{m} \times G_{0} .
$$

It follows from $L^{(2)}=L$ that 


$$
x_{0}=\sum_{j=1}^{n} \beta_{j} x_{j} \in \mathbb{R}^{m} \times G_{0} .
$$

It is obvious that $\delta\left(\mathbb{R}^{m} \times G_{0}\right)=\mathbb{R}^{m} \times G_{0}$ for any $\delta \in \operatorname{Aut}(X)$. So $\beta_{1}^{-1} x_{0} \in$ $\mathbb{R}^{m} \times G_{0}$. Put $x_{1}^{\prime}=x_{1}-\beta_{1}^{-1} x_{0}, x_{j}^{\prime}=x_{j}, j=2, \ldots, n$. Then

$$
\widehat{\mu}_{j}(y)=\left(x_{j}^{\prime}, y\right), \quad y \in L, j=1, \ldots, n .
$$

Moreover,

$$
\sum_{j=1}^{n} \beta_{j} x_{j}^{\prime}=0 .
$$

Put $\mu_{j}^{\prime}=E_{-x_{j}^{\prime}} * \mu_{j}$. Equality (13) implies that the characteristic functions $\widehat{\mu}_{j}^{\prime}(y)=\left(-x_{j}^{\prime}, y\right) \widehat{\mu}_{j}(y)$ satisfy (1). By Lemma 1 , if $\xi_{j}^{\prime}$ are independent random variables with values in $X$ and distributions $\mu_{j}^{\prime}$, then the conditional distribution of $L_{2}^{\prime}=\beta_{1} \xi_{1}^{\prime}+\cdots+\beta_{n} \xi_{n}^{\prime}$ given $L_{1}^{\prime}=\alpha_{1} \xi_{1}^{\prime}+\cdots+\alpha_{n} \xi_{n}^{\prime}$ is symmetric. It follows from (12) that

$$
\widehat{\mu}_{j}^{\prime}(y)=1, \quad y \in L, j=1, \ldots, n .
$$

Hence, $\sigma\left(\mu_{j}^{\prime}\right) \subset A(X, L)=\mathbb{R}^{m} \times G_{0}$. Corollary 1 is proved.

REMARK 1. Corollary 1 implies the following statement (with the same notation). If the conditional distribution of $L_{2}=\beta_{1} \xi_{1}+\cdots+\beta_{n} \xi_{n}$ given $L_{1}=\alpha_{1} \xi_{1}+\cdots+\alpha_{n} \xi_{n}$ is symmetric, then studying the possible distributions $\mu_{j}$ one can suppose without loss of generality that $G=G_{0}$, i.e. the group $G$ itself consists of compact elements.

3. Proof of Theorem 1. To prove Theorem 1 we need some lemmas.

Lemma 4 ([3]). Let $X$ be a finite Abelian group with $X_{(2)}=\{0\}$. Assume that $\alpha_{1}, \alpha_{2}, \beta_{1}, \beta_{2} \in \operatorname{Aut}(X)$ satisfy $\beta_{1} \alpha_{1}^{-1} \pm \beta_{2} \alpha_{2}^{-1} \in \operatorname{Aut}(X)$. Let $\xi_{1}, \xi_{2}$ be independent random variables with values in $X$ and distributions $\mu_{1}, \mu_{2}$. Then the symmetry of the conditional distribution of $L_{2}=\beta_{1} \xi_{1}+\beta_{2} \xi_{2}$ given $L_{1}=\alpha_{1} \xi_{1}+\alpha_{2} \xi_{2}$ implies that $\mu_{1}, \mu_{2} \in I(X)$.

Lemma 5. Let $X$ be a locally compact Abelian group, $K$ a compact subgroup of $X, L=A(Y, K)$, and $\alpha \in \operatorname{Aut}(X)$. Then the following statements are equivalent:

(i) $\alpha(K) \supset K$;

(ii) if $\widetilde{\alpha} y \in L$, then $y \in L$.

Corollary 2. Under the conditions of Lemma 5 the following statements are equivalent:

(i) $\alpha(K)=K$;

(ii) $\widetilde{\alpha}(L)=L$. 
The proofs of Lemma 5 and Corollary 2 are standard and we omit them.

Lemma 6. Let $X$ be a countable discrete torsion Abelian group such that $X_{(2)}=\{0\}$. Assume that $\alpha_{j}, \beta_{j} \in \operatorname{Aut}(X)$ satisfy $\beta_{i} \alpha_{i}^{-1} \pm \beta_{j} \alpha_{j}^{-1} \in \operatorname{Aut}(X)$ for all $i \neq j$. Let $\xi_{1}, \ldots, \xi_{n}, n \geq 2$, be independent random variables with values in $X$ and distributions $\mu_{j}$ such that $\widehat{\mu}_{j}(y) \geq 0$. If the conditional distribution of $L_{2}=\beta_{1} \xi_{1}+\cdots+\beta_{n} \xi_{n}$ given $L_{1}=\alpha_{1} \xi_{1}+\cdots+\alpha_{n} \xi_{n}$ is symmetric, then $\sigma\left(\mu_{j}\right) \subset F$ for all $j$, where $F$ is a finite subgroup of $X$.

Proof. Set $Y=X^{*}$ and note that $Y$ is a totally disconnected compact Abelian group. The compactness of $Y$ implies that $\overline{Y^{(2)}}=Y^{(2)}$. Since $X_{(2)}=\{0\}$, we have $\overline{Y^{(2)}}=Y^{(2)}=Y$, and hence $f_{2}: Y \rightarrow Y$ is an open homomorphism. Put $f_{j}(y)=\widehat{\mu}_{j}(y)$. We restrict ourselves to the case $n=2$. Reasoning as in the proof of Proposition 1 we come to equation (9) for the function $\psi_{1}(y)=-\ln f_{1}(y)$ in a neighbourhood $W$ of zero in $Y$. Since $Y$ is a totally disconnected compact group, there is an open subgroup $H$ of $Y$ such that $H \subset W([5, \S 24.6])$. Since $H$ is an open subgroup, it is also closed and hence compact. By Lemma 2, $\psi_{1}(y)=0$ on $H$. This implies that $f_{1}(y)=1$ for $y \in H$. Thus, $\sigma\left(\mu_{1}\right) \subset A(X, H)=F_{1}$. Since $H$ is an open subgroup its annihilator $F_{1}$ is compact, and as $X$ is discrete, $F_{1}$ is finite. For $\mu_{2}$ we reason similarly. Denote by $F$ the subgroup of $X$ generated by $F_{1}$ and $F_{2}$. Lemma 6 is proved.

Lemma 7. Let $X$ be a locally compact Abelian separable metric group, and let $\xi_{1}, \xi_{2}$ be independent random variables with values in $X$ and distributions $\mu_{1}=m_{K_{1}}, \mu_{2}=m_{K_{2}}$, where $K_{1}, K_{2}$ are finite subgroups of $X$. If $f_{2}, \delta, I \pm \delta \in \operatorname{Aut}(X)$, then the symmetry of the conditional distribution of $L_{2}=\xi_{1}+\delta \xi_{2}$ given $L_{1}=\xi_{1}+\xi_{2}$ implies that $K_{1}=K_{2}=K$ and $\delta(K)=K$.

Proof. Set $Y=X^{*}, f(y)=\widehat{m}_{K_{1}}(y), g(y)=\widehat{m}_{K_{2}}(y), \varepsilon=\widetilde{\delta}, a=I-\varepsilon$, $b=I+\varepsilon, c=a b^{-1}$. Then $c=\widetilde{\gamma}$, where $\gamma=(I+\delta)^{-1}(I-\delta)$. By Lemma 1 , the symmetry of the conditional distribution of $L_{2}$ given $L_{1}$ implies that the characteristic functions $f(y)$ and $g(y)$ satisfy equation (1), which takes the form

$$
f(u+v) g(u+\varepsilon v)=f(u-v) g(u-\varepsilon v), \quad u, v \in Y .
$$

Substituting $v=-u$ in (14) we obtain

$$
g(a u)=f(2 u) g(b u), \quad u \in Y .
$$

This implies that

$$
g(c y)=f\left(2 b^{-1} y\right) g(y), \quad y \in Y .
$$

Put $H_{j}=A\left(Y, K_{j}\right), j=1,2$. It follows from (15) that if $c y \in H_{2}$, then $y \in H_{2}$. By Lemma 5 , this implies that $\gamma\left(K_{2}\right) \supset K_{2}$. Since $K_{2}$ is finite,

$$
\gamma\left(K_{2}\right)=K_{2} \text {. }
$$


We observe that $I+\gamma=2(I+\delta)^{-1}, I-\gamma=2 \delta(I+\delta)^{-1}$. Inasmuch as $f_{2} \in \operatorname{Aut}(X)$, we have $I \pm \gamma \in \operatorname{Aut}(X)$ and $\delta=(I-\gamma)(I+\gamma)^{-1}$. It follows from (16) that $\delta\left(K_{2}\right)=K_{2}$, and by Corollary $2, \varepsilon\left(H_{2}\right)=H_{2}$. Consider the restriction of equation (14) to the subgroup $H_{2}$. We have

$$
f(u+v)=f(u-v), \quad u, v \in H_{2} .
$$

Hence,

$$
f(2 y)=1, \quad y \in H_{2} .
$$

Since $f_{2} \in \operatorname{Aut}(X)$ and $K_{2}$ is a finite group, we conclude that $\left(K_{2}\right)^{(2)}=K_{2}$, and by Corollary $2,\left(H_{2}\right)^{(2)}=H_{2}$. It follows from $(17)$ that $f(y)=1$ for $y \in H_{2}$, and hence $H_{2} \subset H_{1}$. Reasoning similarly we deduce that (14) implies $\varepsilon\left(H_{1}\right)=H_{1}$ and

$$
g(2 \varepsilon y)=1, \quad y \in H_{1},
$$

so that $H_{1} \subset H_{2}$. Thus, $H_{1}=H_{2}=H, K_{1}=K_{2}=K$. Since $\varepsilon(H)=H$, by Corollary $2, \delta(K)=K$. Lemma 7 is proved.

Now we can prove Theorem 1.

Proof of Theorem 1. Set $Y=X^{*}$. The necessity of the condition $X_{(2)}$ $=\{0\}$ follows from the fact that if $\xi_{j}$ are arbitrary independent random variables with values in $X_{(2)}$ and $\alpha_{j}, \beta_{j} \in \operatorname{Aut}(X)$, then the conditional distribution of $L_{2}=\beta_{1} \xi_{1}+\cdots+\beta_{n} \xi_{n}$ given $L_{1}=\alpha_{1} \xi_{1}+\cdots+\alpha_{n} \xi_{n}$ is symmetric (see in more detail [3, Remark 1]). Let us prove the sufficiency.

Considering the new independent random variables $\xi_{1}^{\prime}=\alpha_{1} \xi_{1}, \xi_{2}^{\prime}=\alpha_{2} \xi_{2}$, we can assume from the beginning that $L_{1}=\xi_{1}+\xi_{2}, L_{2}=\delta_{1} \xi_{1}+\delta_{2} \xi_{2}$, where $\delta_{1}, \delta_{2}, \delta_{1} \pm \delta_{2} \in \operatorname{Aut}(X)$. By Lemma 1 , the symmetry of the conditional distribution of $L_{2}$ given $L_{1}$ implies that the characteristic functions $\widehat{\mu}_{j}$ satisfy equation (3) which takes the form

$$
\widehat{\mu}_{1}\left(u+\widetilde{\delta}_{1} v\right) \widehat{\mu}_{2}\left(u+\widetilde{\delta}_{2} v\right)=\widehat{\mu}_{1}\left(u-\widetilde{\delta}_{1} v\right) \widehat{\mu}_{2}\left(u-\widetilde{\delta}_{2} v\right), \quad u, v \in Y .
$$

Put $\nu_{j}=\mu_{j} * \bar{\mu}_{j}, j=1,2$. Then $\widehat{\nu}_{j}(y)=\left|\widehat{\mu}_{j}(y)\right|^{2} \geq 0$. Set $f(y)=\widehat{\nu}_{1}(y)$, $g(y)=\widehat{\nu}_{2}(y), \delta=\delta_{1}^{-1} \delta_{2}, \varepsilon=\widetilde{\delta}$. In this notation equation (18) is transformed into (14). We will prove Theorem 1 if we verify that the functions $f(y)$ and $g(y)$ take on the values 0 and 1 only.

By Remark 1, we can suppose from the beginning that $X$ is a torsion group. Put $L=\{y \in Y: f(y)=1\}, H=\{y \in Y: g(y)=1\}, K=A(X, L)$, $G=A(X, H)$. By Lemma $6, \sigma\left(\nu_{j}\right) \subset F, j=1,2$, where $F$ is a finite subgroup of $X$. It is obvious that $K$ and $G$ must also be finite subgroups because $K, G \subset F$. It follows from (14) that

$$
f^{n}(u+v) g^{n}(u+\varepsilon v)=f^{n}(u-v) g^{n}(u-\varepsilon v), \quad u, v \in Y,
$$


for any natural $n$. It is clear that the limits

$$
\lim _{n \rightarrow \infty} f^{n}(y)=\widehat{m}_{K}(y), \quad \lim _{n \rightarrow \infty} g^{n}(y)=\widehat{m}_{G}(y)
$$

exist. Letting $n \rightarrow \infty$ in (19) we see that the functions $\widehat{f}(y)=\widehat{m}_{K}(y)$ and $\widehat{g}(y)=\widehat{m}_{G}(y)$ also satisfy (14). Since $X$ is a torsion group and $X_{(2)}=\{0\}$, we have $f_{2} \in \operatorname{Aut}(X)$ and so we can apply Lemma 7 . We obtain $K=G$, $L=H$ and $\delta(K)=K$. By Corollary 2,

$$
\varepsilon(L)=L .
$$

This implies that the homomorphism induced by $\varepsilon$ on $Y / L$ is an automorphism. Moreover, it follows from $L=H$ that $f(y)=g(y)=1$ for $y \in L$. Hence, $f$ and $g$ are $L$-invariant. Therefore (14) induces an equation on $Y / L$. Since $Y / L \approx K^{*}$ and $K$ is a finite subgroup with $K_{(2)}=\{0\}$, we can apply Lemma 4 to complete the proof of Theorem 1.

We add to Theorem 1 the following statement.

Proposition 2. Let $X$ be a locally compact Abelian separable metric group, and let $\xi_{1}, \xi_{2}$ be independent random variables with values in $X$ and distributions $\mu_{1}=\mu_{2}=m_{K}$, where $K$ is a compact subgroup of $X$. Assume that $\delta, I \pm \delta \in \operatorname{Aut}(X)$. Set $\gamma=(I+\delta)^{-1}(I-\delta)$. Then the following statements are equivalent:

(i) the conditional distribution of $L_{2}=\xi_{1}+\delta \xi_{2}$ given $L_{1}=\xi_{1}+\xi_{2}$ is symmetric;

(ii) $\gamma(K) \supset K$.

Proof. Set $\varepsilon=\widetilde{\delta}, a=I-\varepsilon, b=I+\varepsilon, c=a b^{-1}$. Then $c=\widetilde{\gamma}$. Assume that (i) holds. Put $L=A(Y, K), f(y)=\widehat{m}_{K}(y)$. By Lemma $1, f$ satisfies (1), which takes the form

$$
f(u+v) f(u+\varepsilon v)=f(u-v) f(u-\varepsilon v), \quad u, v \in Y .
$$

Substituting $v=-u$ we find

$$
f(a u)=f(2 u) f(b u), \quad u \in Y .
$$

Hence,

$$
f(c y)=f\left(2 b^{-1} y\right) f(y), \quad y \in Y .
$$

Since

$$
f(y)= \begin{cases}1, & y \in L \\ 0, & y \notin L\end{cases}
$$

equation (22) implies that if $c y \in L$, then $y \in L$. Now Lemma 5 yields (ii).

Conversely, assume that (ii) holds. We will verify that $f$ satisfies (21), which, by Lemma 1, proves (i). Note that by Lemma 5, (ii) is equivalent to the statement: if $c y \in L$, then $y \in L$. Suppose that for some $u, v \in Y$ the 
left-hand side of (21) is equal to 1 . Then

$$
u+v, u+\varepsilon v \in L .
$$

This implies that $a v \in L$. Inasmuch as $a v=c b v$, we have $c b v \in L$, and hence

$$
b v=(I+\varepsilon) v \in L .
$$

It follows from (23) and (24) that $u-v, u-\varepsilon v \in L$, i.e. the right-hand side of $(21)$ is 1 . We verify similarly that if the right-hand side of $(21)$ is 1 , then the same is true for the left-hand side. Proposition 2 is proved.

REMARK 2. It follows from the proof of Theorem 1 that if $X$ is a countable discrete Abelian group such that $X_{(2)}=\{0\}, Y=X^{*}$ and $\varepsilon, I \pm \varepsilon \in \operatorname{Aut}(Y)$, then all solutions of (14) in the class of characteristic functions are of the form

$$
f(y)=\left(x_{1}, y\right) \widehat{m}_{K}(y), \quad g(y)=\left(x_{2}, y\right) \widehat{m}_{K}(y),
$$

where $x_{1}, x_{2} \in X$ and $K$ is a finite subgroup of $X$ with $\delta(K)=K, \delta=\widetilde{\varepsilon}$.

Remark 3. Let $X$ be a countable discrete Abelian group. Assume that $\alpha_{j}, \beta_{j} \in \operatorname{Aut}(X)$ satisfy $\beta_{i} \alpha_{i}^{-1} \pm \beta_{j} \alpha_{j}^{-1} \in \operatorname{Aut}(X)$ for all $i \neq j$. Let $\xi_{1}, \ldots, \xi_{n}$, $n \geq 2$, be independent random variables with values in $X$ and distributions $\mu_{j}$ with non-vanishing characteristic functions. If the conditional distribution of $L_{2}=\beta_{1} \xi_{1}+\cdots+\beta_{n} \xi_{n}$ given $L_{1}=\alpha_{1} \xi_{1}+\cdots+\alpha_{n} \xi_{n}$ is symmetric, then $\sigma\left(\mu_{j}^{\prime}\right) \subset X_{(2)}$ for all $j$, for some shifts $\mu_{j}^{\prime}$ of the distributions $\mu_{j}$.

We shall restrict ourselves to the proof for the case $n=2$. Clearly we can assume that $L_{1}=\xi_{1}+\xi_{2}, L_{2}=\xi_{1}+\delta \xi_{2}$, where $\delta, I \pm \delta \in \operatorname{Aut}(X)$. Put $\nu_{j}=\mu_{j} * \bar{\mu}_{j}, j=1,2$. It is easily seen that our statement will be proved if we verify that $\sigma\left(\nu_{j}\right) \subset X_{(2)}$. We note that $\widehat{\nu}_{j}(y)>0$ for all $Y$. Put $f(y)=\widehat{\nu}_{1}(y), g(y)=\widehat{\nu}_{2}(y)$. Reasoning as in the proof of Proposition 1 we arrive at equation (8) for the function $\psi_{1}(y)=-\ln \widehat{\nu}_{1}(y)$ for all $u, v \in Y$. It follows that $\psi_{1}$ satisfies (9) on the subgroup $Y^{(2)}$. Since $Y^{(2)}$ is compact, by Lemma $2, \psi_{1}(y)=0$ for $y \in Y^{(2)}$. This implies that $\sigma\left(\nu_{1}\right) \subset A\left(X, Y^{(2)}\right)=$ $X_{(2)}$. Reasoning similarly we prove that $\sigma\left(\nu_{2}\right) \subset X_{(2)}$.

4. Heyde theorem for $X=\mathbb{R} \times G$, where $G$ is a countable discrete Abelian group with $G_{(2)}=\{0\}$. We will use Theorem 1 to prove the following statement.

Theorem 2. Let $X=\mathbb{R} \times G$, where $G$ is a countable discrete Abelian group such that $G_{(2)}=\{0\}$. Assume that $\alpha_{1}, \alpha_{2}, \beta_{1}, \beta_{2} \in \operatorname{Aut}(X)$ satisfy $\beta_{1} \alpha_{1}^{-1} \pm \beta_{2} \alpha_{2}^{-1} \in \operatorname{Aut}(X)$. Let $\xi_{1}, \xi_{2}$ be independent random variables with values in $X$ and distributions $\mu_{1}, \mu_{2}$. If the conditional distribution of $L_{2}=$ $\beta_{1} \xi_{1}+\beta_{2} \xi_{2}$ given $L_{1}=\alpha_{1} \xi_{1}+\alpha_{2} \xi_{2}$ is symmetric, then $\mu_{j}=\lambda_{j} * \pi_{j}$, where $\lambda_{j}$ are Gaussian distributions on $\mathbb{R}$, and $\pi_{j} \in I(X), j=1,2$. 
To prove Theorem 2 we need the following

Lemma 8 ([3]). The conclusion of Theorem 2 is true when $G$ is a finite Abelian group.

Proof of Theorem 2. We have $Y=X^{*} \approx \mathbb{R} \times H$, where $H=G^{*}$. To simplify notation we assume that $Y=\mathbb{R} \times H$. Reasoning as in the proof of Theorem 1 we reduce the proof to the case when $L_{1}=\xi_{1}+\xi_{2}, L_{2}=\xi_{1}+\delta \xi_{2}$ and $\delta, I \pm \delta \in \operatorname{Aut}(X)$. We need to solve equation (14), where $f(y)=\widehat{\mu}_{1}(y)$, $g(y)=\widehat{\mu}_{2}(y), \varepsilon=\widetilde{\delta}$. By Remark 1 we can assume that $G$ is a torsion group. This implies that $H$ is a totally disconnected compact Abelian group. Denote elements of $Y$ by $(s, h), s \in \mathbb{R}, h \in H$. It is obvious that if $d \in \operatorname{Aut}(Y)$, then $d(\mathbb{R})=\mathbb{R}$ and $d(H)=H$. We will retain notation $d$ for the restrictions of $d$ to $\mathbb{R}$ and to $H$. For this reason we write $d(s, h)=(d s, d h),(s, h) \in Y$. In this notation equation (14) takes the form

$$
\begin{aligned}
& f\left(s+s^{\prime}, h+h^{\prime}\right) g\left(s+\varepsilon s^{\prime}, h+\varepsilon h^{\prime}\right) \\
& \quad=f\left(s-s^{\prime}, h-h^{\prime}\right) g\left(s-\varepsilon s^{\prime}, h-\varepsilon h^{\prime}\right), \quad(s, h),\left(s^{\prime}, h^{\prime}\right) \in Y .
\end{aligned}
$$

Substituting $s=s^{\prime}=0$ in (25) we come to the equation

$$
f\left(0, h+h^{\prime}\right) g\left(0, h+\varepsilon h^{\prime}\right)=f\left(0, h-h^{\prime}\right) g\left(0, h-\varepsilon h^{\prime}\right), \quad h, h^{\prime} \in H .
$$

It follows from Remark 2 that all solutions of (26) are of the form

$$
f(0, h)=\left(g_{1}, h\right) \widehat{m}_{K}(h), \quad g(0, h)=\left(g_{2}, h\right) \widehat{m}_{K}(h), \quad h \in H,
$$

where $K$ is a finite subgroup of $G, g_{1}, g_{2} \in G$ and $\delta(K)=K$. Put $B=$ $A(H, K)$. Substitute (27) into (26) and consider the resulting equation on $B$. We obtain

$$
2\left(g_{1}+\delta g_{2}\right) \in K
$$

Since $K$ is a finite group and $G_{(2)}=\{0\}$, this implies that

$$
g_{1}+\delta g_{2} \in K \text {. }
$$

Put $\mu_{1}^{\prime}=E_{\delta g_{2}} * \mu_{1}, \mu_{2}^{\prime}=E_{-g_{2}} * \mu_{2}$. It follows from (29) that the characteristic functions $\widetilde{\mu}_{1}^{\prime}(s, h)$ and $\widetilde{\mu}_{2}^{\prime}(s, h)$ satisfy $(25)$. Note that

$$
\widetilde{\mu}_{1}^{\prime}(0, h)=\widetilde{\mu}_{2}^{\prime}(0, h)= \begin{cases}1, & h \in B, \\ 0, & h \notin B .\end{cases}
$$

It follows that the characteristic functions $\widetilde{\mu}_{1}^{\prime}(s, h)$ and $\widetilde{\mu}_{2}^{\prime}(s, h)$ are $B$ invariant. Hence, $\sigma\left(\mu_{j}\right) \subset A(X, B)=\mathbb{R} \times K$. Since $\delta(\mathbb{R} \times K)=\mathbb{R} \times K$, we can apply Lemma 8 and complete the proof of Theorem 2 .

REMARK 4. The assertion of Theorem 2 is also valid for the group $X=$ $\mathbb{R}^{m} \times G$, where $m>1$, and $G$ is a countable discrete Abelian group such that $G_{(2)}=\{0\}$. To prove this we reason as in the proof of Theorem 2 and reduce the proof to the case when $G$ is a finite group. The proof in [3] for the case of $G$ finite is based on Theorem A. This proof remains valid when $m>1$, 
but instead of Theorem A we need the following statement: Let $X=\mathbb{R}^{m}$, where $m>1$. Assume that $\alpha_{j}, \beta_{j} \in \operatorname{Aut}(X)$ satisfy $\beta_{i} \alpha_{i}^{-1} \pm \beta_{j} \alpha_{j}^{-1} \in \operatorname{Aut}(X)$ for all $i \neq j$. Let $\xi_{1}, \ldots, \xi_{n}, n \geq 2$, be independent random variables with values in $X$ and distributions $\mu_{j}$. If the conditional distribution of $L_{2}=$ $\beta_{1} \xi_{1}+\cdots+\beta_{n} \xi_{n}$ given $L_{1}=\alpha_{1} \xi_{1}+\cdots+\alpha_{n} \xi_{n}$ is symmetric, then all $\mu_{j}$ are Gaussian. To prove this, we reason as in the proof of Proposition 1. We retain the same notation and restrict ourselves to the case $n=2$. We arrive at equation (9) in a neighbourhood $W$ of zero in $Y=\mathbb{R}^{m}$. Hence, $\psi_{1}(s)$ is an ordinary polynomial in $W$. By Lemma 3 , the representation

$$
f_{1}(s)=e^{-\psi_{1}(s)}, \quad s \in W,
$$

can be extended from $W$ to $\mathbb{R}^{m}$. Standard arguments involving the Marcinkiewicz theorem $([8$, Ch. $1, \S 5])$ and the Cramer theorem on decomposition of a Gaussian distribution in $\mathbb{R}^{m}$ show that $\mu_{1}$ is Gaussian. For $\mu_{2}$ we reason similarly.

\section{References}

[1] G. M. Feldman, Marcinkiewicz and Lukacs theorems on Abelian groups, Theory Probab. Appl. 34 (1989), 290-297.

[2] - Arithmetic of Probability Distributions and Characterization Problems on Abelian Groups, Amer. Math. Soc. Transl. Math. Monogr. 116, Providence, RI, 1993.

[3] - On the Heyde theorem for finite Abelian groups, J. Theoret. Probab. 17 (2004), 929-941.

[4] -, On a characterization theorem for locally compact Abelian groups, Probab. Theory Related Fields 133 (2005), 345-357.

[5] E. Hewitt and K. A. Ross, Abstract Harmonic Analysis, Vol. 1, Springer, Berlin, 1963.

[6] C. C. Heyde, Characterization of the normal low by the symmetry of a certain conditional distribution, Sankhyā Ser. A 32 (1970), 115-118.

[7] A. M. Kagan, Yu. V. Linnik and C. R. Rao, Characterization Problems of Mathematical Statistics, Wiley, New York, 1973.

[8] Ju. V. Linnik and I. V. Ostrovskiŭ, Decomposition of Random Variables and Vectors, Amer. Math. Soc. Transl. Math. Monogr. 48, Providence, RI, 1977.

[9] K. R. Parthasarathy, Probability Measures on Metric Spaces, Academic Press, New York, 1967.

Mathematical Division

B. Verkin Institute for Low Temperature Physics and Engineering

National Academy of Sciences of Ukraine

47, Lenin Ave., Kharkov, 61103, Ukraine

E-mail: feldman@ilt.kharkov.ua 